\title{
Programas de intervención psicosocial en sistema penitenciario cerrado con agresores sexuales: Una aproximación a la situación en Chile
}

\section{Psychosocial intervention programs targeting imprisoned sex offenders: An approach to the chilean situation}

\begin{abstract}
Gonzalo Javier Lira Mendiguren. Escuela de Psicología Universidad de Valparaíso. Magíster en Psicología Social, mención Psicología Jurídica. Coinvestigador Núcleo de Investigación Delitos Sexuales Población Carcelaria PMI-MECESUP Universidad de Valparaíso. Avenida Brasil, N²140, Valparaíso,VRegión, Chile.gonzalo.lira@uv.cl

Carlos Varas Alfaro. Escuela de Psicología, Universidad de Valparaíso. Magíster en Psicología Integrativa, Universidad Adolfo Ibáñez/ICPSI. Investigador asociado Núcleo de Investigación Delitos Sexuales Población Carcelaria PMI-MECESUPUniversidad de Valparaíso. Avenida Brasil, N²140, Valparaíso, V Región, Chile.carlos.varas@uv.cl

Sara Salum Alvarado. Escuela de Trabajo Social Universidad de Valparaíso. Doctora en Estudios Americanos, Master International Social Work, criminóloga. Directora de Investigación Núcleo Delitos Sexuales Población Carcelaria PMI-MECESUP U. de Valparaíso. Colón N²128 Valparaíso, V Región Chile. Sara.salum@uv.cl

Elena Salum Alvarado. Escuela de Trabajo Social Universidad de Valparaíso. Doctora en Estudios Americanos, Magíster en Gestión y Política Pública Universidad de Chile. Coinvestigadora Núcleo Delitos Sexuales Población Carcelaria PMI-MECESUP U. de Valparaíso. Colón No2128 Valparaíso,V región Chile. Elena.salum@uv.cl
\end{abstract}

\begin{abstract}
Resumen
A partir de la revisión de las distintas experiencias internacionales de programas de intervención psicosocial en agresores sexuales privados de libertad (sistema cerrado), se analiza y describe la situación del sistema carcelario cerrado en Chile, concluyendo que la aplicación de experiencias en la temática resulta escasa y asistemática. Esto evidencia la falta de una política criminal clara destinada a la población de agresores sexuales, surgiendo la necesidad de establecer lineamientos claros de intervención a nivel del sistema carcelario.

Palabras claves. Agresor sexual, programa de intervención psicosocial, política criminal.

Abstract

Based in a review of the various international psychosocial intervention programs focusing on incarcerated sexual offenders, the Chilean experience is examined. The implementation of psychosocial interventions targeting sexual offenders in Chile is scarce and unsystematic. Results highlight lack of a clear criminal policy addressing sex offending inmate population. Clear guidelines for this type of interventions within the Chilean Prison system need to be developed.
\end{abstract}

Key words. Sexual offenders, Psychosocial intervention programs, Prison System, Criminal Policy.

\section{Delitos sexuales en cifras}

La delincuencia y la conducta criminal se han constituido en materias que generan interés tanto a nivel de la opinión pública como en el campo académico, debido a su complejidad, siendo la Criminología la disciplina científica que ha profundizado en esta temática. Centra su interés en el delito, en el individuo que delinque, en el control social y la víctima. Surge como una ciencia integral que estudia el comportamiento delictivo y la reacción social frente al mismo, y comparte algunos conocimientos, términos e instrumentos de investigación con otras disciplinas, como la sociología, la psicología, la medicina, la biología y el derecho (Garrido, Stangeland \& Redondo, 1999).

Dentro de la delincuencia violenta, destaca la de carácter sexual, por suponer un fuerte atropello a la libertad de las personas. Las agresiones sexua- 
les constituyen un determinado tipo de parafilias -conductas sexuales poco comunes o extrañas- que tienen la peculiaridad de suponer una transgresión del libre consentimiento de otra persona (Echeburúa, 1994). Jorge Barudy (1999) plantea que estos delitos constituyen un profundo y grave atentado a la integridad física o psicológica de las víctimas, comparable a una "tentativa de asesinato moral". En la literatura, el delito sexual es asociado a características psicológicas, a diferencia de lo que ocurre con los delitos comunes, los que se asocian a explicaciones ambientalistas y sociales.

De acuerdo a la Fiscalía Nacional, el año 2012 se vio marcado por el aumento de denuncias de delitos sexuales. En esta categoría, 13.540 denuncias correspondieron a imputados conocidos y 9.243 a imputados desconocidos, lo que, en total, sumó 22.783 ingresos durante el 2012. Esta situación implica un aumento de un 7,6\%, es decir, 1.607 casos más que en 2011. Hubo 1.079 juicios orales por este tipo de delitos, siendo las regiones de Los Ríos, Magallanes, El Maule y Los Lagos las que registraron la mayor cantidad de estos juicios.

En 2013, el "abuso sexual de menor de 14 años" fue un ilícito que evidenció un fuerte incremento, de $31,5 \%$, es decir, de 4.970 denuncias se subió a una cifra de 6.537 denuncias. Y fueron alrededor de 500 establecimientos educacionales los que presentan investigaciones de este tipo. Esta cifra incluye también a personas que tienen alguna relación tangencial con los colegios, como vendedores o movilizadores.

Los delitos sexuales contra menores de edad abarcan un amplio espectro de conductas, por lo cual la Fiscalía Nacional evidenció la necesidad de avanzar en materia legislativa, a fin de poder abordar integralmente el fenómeno, de tal forma que los órganos del Estado puedan otorgar una respuesta holística a este delito, situación que no se da en la práctica, ya que en materia de delitos sexuales se presentó un total de 16 proyectos de Ley ante el Congreso Nacional en el año 2012.

Frente a estas iniciativas, la Fiscalía Nacional plantea refundir los actuales proyectos que se tramitan ante el Congreso y darles un tratamiento orgánico, con una mirada integral. En materia de Código Penal, los proyectos de ley dicen relación con el aumento de penas, la imprescriptibilidad de los delitos sexuales, circunstancias modificatorias, la ampliación de inhabilidades y modificaciones de algunos tipos penales.

En materia de penas sustitutivas conforme a la ley 18.216, la Fiscalía Nacional propone su modifi- cación en orden a restringir su aplicación en caso de condenados por delitos sexuales, de tal manera de garantizar la adecuada protección de las víctimas por este tipo de delitos. En esta misma línea, se estima del todo necesarias las restricciones a la eliminación de las anotaciones penales respecto de estos condenados (Fiscalía Nacional, 2013).

Según datos de Carabineros de Chile (AUPOL), en 2013 las detenciones por abusos sexuales a nivel país presentaron una frecuencia absoluta de 1.087 casos, en tanto que las denuncias correspondieron a 7.049 casos. Si analizamos las tasas de denuncias, las detenciones corresponden a 6,2 casos cada 100.000 habitantes, en tanto que las denuncias corresponden a una tasa de 40,1 cada 100.000 habitantes.

Según la misma fuente, en materia de violaciones se realizaron 440 detenciones, en tanto que las denuncias ascendieron a 1.834 casos. En este ámbito, las detenciones corresponden a una tasa de 2,5 cada 100.000 habitantes, en tanto que las denuncias correspondieron a una tasa de 10,4.

Es así que las cifras anteriores dan cuenta de la relevancia del problema y la necesidad de que el Estado pueda dar respuesta a través de una política criminal especializada a los agresores sexuales.

\section{Caracterización de los agresores sexuales}

Si bien no es posible señalar que exista una homogeneidad en cuanto a las características de los agresores o delincuentes sexuales, sí es posible señalar que existen diversas tipologías o taxonomías respecto de estos. Autores como Echeburúa y Guerricaechevarría (2000), Barudy (1999) y Garrido (1993) señalan que estos pertenecen en mayor proporción al sexo masculino, presentan una asociación a un mayor impulso sexual y agresividad, trastornos de la identidad, la autoestima y las capacidades para establecer y mantener relaciones interpersonales y una tendencia a la distorsión. Esto último puede constituir una de las características más marcadas en los agresores sexuales, dado que esto permitiría explicar un estilo de funcionamiento, se autogenera un ideal de situación que desea con insistencia y adecúa su comportamiento a una pseudoverdad, rebasando normas sociales y estableciendo sus propios criterios basados en sus deseos.

Otras características son las dificultades para establecer relaciones íntimas, emocionales y sexuales. El delincuente sexual es descrito como una persona que experimenta impotencia y falta de asertividad con las mujeres, junto a un déficit en el control de su impulso sexual (Beneyto y Garrido 1997). 
En una propuesta complementaria, se destacan tres áreas de carencias diferentes en este grupo de sujetos, pero interrelacionadas entre sí: en el comportamiento y preferencia sexual, en la conducta social más amplia y en sus cogniciones ("distorsiones cognitivas") (Berlin, 2000; Brown, 2005; Echeburúa y Guerricaecheverría, 2000; Marshall, 2001; Redondo, 2002).

Existen algunas tipologías de delincuentes sexuales, pudiendo ser agrupadas con base en: a.- Tipo de agresión sexual; b.- Tipo de víctima; c.- Tipo de relación existente con la víctima; y, d.- Mecanismos psicológicos a la base de dicha conducta (Calderón, Duguet, Espinoza \& Fuentealba, 2001)

También pueden ser clasificados con base en la relación existente entre la víctima y el agresor, pudiéndose identificar: a.- agresores sexuales pertenecientes al ámbito familiar (a su vez presenta dos subcategorías: a1.- victima adulta, a2.- victima menor de edad) (Calderón, Duguet, Espinoza \& Fuentealba, 2001).

Cada uno de estas tipologías se diferencian en cuanto a estrategia de acercamiento: encantamiento, amedrentamiento, agresividad y violencia, móvil, y a su vez tiene en común un agresor con escasas habilidades sociales, inmadurez afectiva y bajo nivel de autoestima.

En los casos de delincuentes sexuales, Gómez (1999) sostiene que es posible realizar tratamiento psicológico para producir una mejoría en su estabilidad psíquica, considerando que si el agresor posee recursos intelectuales y culturales más elevados, es capaz de modificar los criterios alterados a nivel cognitivo, si bien el cambio a nivel emocional y comportamental es más difícil.

A medida que desciende el nivel de recursos del agresor existe más resistencia a una modificación, aunque solo sea a nivel cognitivo, de las ideas que rigen su concepto de los roles masculino y femenino. Debido a su escasez de recursos, el pensamiento de estos individuos es más rígido y estereotipado que lo normal. Su resistencia al cambio se fundamenta en la imposibilidad de hacer un análisis racional de los principios que han motivado la oposición y persistencia de dichas ideas (Gómez, 1999).

\section{Programas de tratamiento penitenciario de agresores sexuales}

Los agresores sexuales representan un peligro para la sociedad. Si bien el número de reclusos que cumple condena por delitos sexuales es bajo en comparación a otros delitos, sus consecuencias tan- to a nivel de la víctima, su familia, la comunidad y la sociedad en general hacen que estos hechos tengan una connotación social relevante.

Existe una polémica acerca de la reintegración de los agresores sexuales. Algunos especialistas creen que este tipo de agresores no deberían salir de la cárcel hasta que cumplan su sanción penal, no siendo atendible argumentos de buena conducta, dado que esta no es señal objetiva de cambio de conducta o recuperación, e incluso algunos plantean que personas que han agredido sexualmente no tendrían posibilidad de recuperación social. Por otra parte, existen otros especialistas que plantean que la cárcel no sería un lugar adecuado para su recuperación y que esta merma sus posibilidades de lograr la reinserción social y ladisminución de su reincidencia. La aplicación de tratamientos a los delincuentes sexuales es una estrategia relativamente reciente surgida en Norteamérica en la década de 1970 (Canadá y EE.UU.) y limitada a los países desarrollados y -dentro de ellos- a unos cuantos programas que se aplican en algunas prisiones, y en contadas ocasiones en la comunidad. Así, la proporción de agresores sexuales que recibe tratamiento es mínima en comparación al resto de criminalidad que recibe sanción penal en el sistema carcelario (Redondo, 2006).

Tanto el riesgo de reincidencia como las posibilidades de tratamiento van a depender de la tipología del agresor de que se trate y, específicamente, de los factores de riesgo que confluyan en cada sujeto. La investigación más moderna en psicología criminal ha puesto de relieve la existencia tanto de factores de riesgo -que aumentan el riesgo- como de factores de protección o resistencia (entre ellos el hecho de ser hijo primogénito, poseer alta autoestima y autocontrol, haber tenido cuidados alternativos a los paternos en caso de riesgo familiar y haber tenido modelos de apoyo del mismo sexo), los cuales protegen al individuo disminuyendo el riesgo de conducta delictiva (Garrido, 2005).

\section{Experiencias internacionales}

Siguiendo la línea de pensamiento establecida por Budrionis y Jongsma (2003, cit. en Redondo, 2006), el tratamiento de agresores sexuales dispone de buenas estrategias psicológicas, con niveles de desarrollo equivalentes a otros campos de intervención psicológica. No obstante lo anterior, la principal problemática asociada resulta de carácter práctica, ya que la aplicación de tratamientos a los delincuentes sexuales "es una actividad limitada a unos pocos países desarrollados y, dentro de ellos, a unos cuan- 
tos programas en prisiones, y esporádicamente en la propia comunidad" (Redondo, 2006, p. 2).

A continuación se exponen las características más comunes a los programas de intervención con agresores sexuales (Redondo, 2006, p.3):

- Suelen ser programas intensivos de larga duración.

- Incluyen técnicas dirigidas específicamente a las tres áreas problemáticas mencionadas: comportamiento sexual desviado, distorsiones cognitivas $\mathrm{y}$ funcionamiento social del sujeto.

- A veces se utilizan, en el contexto global del programa, agentes químicos inhibidores del impulso sexual. Se han utilizado tres medicaciones reductoras del impulso sexual masculino: el acetato deciproterona, el acetato de medroxiprogesterona y, más modernamente, el agonista análogo de la hormona liberadora de la gonadotropina (GnRH) (Greenberg y Bradford, 1997; Marshall y Redondo, 2002; Rösler y Witztum, 2000).

- En la aplicación suelen intervenir diversos terapeutas (con frecuencia hombre y mujer) que entrenan a los agresores sexuales en habilidades sociales específicas con un doble propósito: 1) que aprendan a inhibir las conductas delictivas, y 2) que aprendan las habilidades de comunicación necesarias para establecer relaciones sexuales adultas y consentidas.

- En general, el tratamiento suele tener carácter voluntario, aunque la eventual participación del sujeto en el mismo suele ser recompensada con beneficios penales y penitenciarios (permisos de salida al exterior, mejora del régimen de vida en prisión o concesión futura de la libertad condicional).

A continuación se presenta un breve análisis sobre experiencia comparada en materia de intervención en agresores sexuales en situación intramuro de cumplimiento de condena.

\section{Experiencia canadiense}

Un ejemplo de prevención terciaria son los programas impulsados por el Servicio Correccional de Canadá. Este servicio considera para tratamiento a cualquier sujeto que: a) sea declarado culpable de una ofensa sexual, b) sea declarado culpable de un crimen motivado sexualmente, c) haya admitido haber realizado una agresión sexual, aun cuando no se haya declarado culpable.

Estos sujetos son evaluados y luego se les diseña un tratamiento basado en la identificación de la natu- raleza del modelo de conducta de cada sujeto, junto con las estrategias necesarias para influir en la reducción del riesgo de reincidencia.

La evaluación se desarrolla a lo largo del cumplimiento de la condena y aplica una variedad de metodologías. Al ingreso se evalúan áreas como: la historia y desarrollo de su conducta sexual, preferencias sexuales, actitudes y distorsiones cognoscitivas, competencia social, historia médica, psicopatología, evaluaciones anteriores y resultados del tratamiento.

Con el diagnóstico se define el tratamiento terapéutico orientado a reducir el riesgo de reincidencia, por medio del tratamiento a las distorsiones cognoscitivas, la excitación anticonvencional, la fantasía, competencia social, manejo de la ira, la empatía, las actitudes hacia la sexualidad, el conocimiento de la víctima y la prevención de la recaída.

El programa es de naturaleza cognoscitiva conductual y grupal, pero tiene una intervención individual cuando es necesario. Por ello, se hace énfasis en que los agresores tomen responsabilidad por sus acciones, reconozcan su ciclo de agresión e identifiquen sus situaciones de alto riesgo para que elaboren sus estrategias de prevención. Esto implica reducir el riesgo de reincidencia sexual por una combinación de autogestión y de control externo.

Junto con la intervención o tratamiento, el sistema de Canadá mantiene una línea de investigación destinada a reforzar y mejorar el trabajo realizado. Este programa propone desarrollar investigación que pueda ser aplicada en las políticas, programas y trato con delincuentes sexuales. Los tipos de investigación se han orientado a: 1) Mejorar la información y la recolección de datos sobre la internación; 2) Ayudar en la toma de decisiones sobre qué programas o tratamiento se necesita antes de la obtención de la libertad; 3) El diseño del programa de integración a la comunidad; 4) La supervisión e información sobre agresores sexuales beneficiados por los diversos tipos de programas; 5) Cómo mejorar la recolección de información cuando se produce la primera salida a la comunidad, y 6) Cómo mejorar el trato de la comunidad cuando se produce el egreso.

\section{Experiencia norteamericana}

Respecto de los agresores sexuales violentos, se pasa de un modelo de internamiento indeterminado en centros psiquiátricos con fines de tratamiento (décadas de 1970 y 1980) a un modelo de pena regida por el principio de proporcionalidad a la que se le adiciona una medida de seguridad regida por el principio de inocuización (una medida de seguridad 
ejecutada con posterioridad a la pena y de duración indeterminada), siendo los requisitos de procedencia: ser condenado por un delito sexual violento, con afectación plural de víctimas (al menos dos personas) y la existencia de un pronóstico de reincidencia futura (selective incapacitation, "Sexually Violent Predators Act" del Estado de Washington, de 1990). Este "sistema dualista" puede completarse con medidas adicionales tales como la castración química, el tratamiento hormonal, las medidas de supervisión y control posteriores al cumplimiento de la condena (supervised release y parole) y los deberes de registro de los exdelincuentes sexuales y notificación al vecindario por medios de comunicación privados o públicos e internet (recuérdese, al respecto, las denominadas "Leyes Megan"). Dicho modelo político-criminal fue adoptado, con más o menos modificaciones, por Inglaterra (Guillamondegui, 2006).

\section{Experiencia española}

El Reglamento Penitenciario de 1996 establece que "la Administración Penitenciaria podrá realizar programas específicos de tratamiento para internos condenados por delitos contra la libertad sexual a tenor de su diagnóstico previo" (Art. 116.4 R.P.).

El ingreso a los mismos será voluntario (como principio rector del tratamiento penitenciario) y no podrá suponer la marginación de los reclusos afectados en las prisiones (a modo de prevención a cualquier tipo de discriminación).

Con motivo de la prescripción legal aludida, la Dirección General de Instituciones Penitenciarias a partir de 1999 empezó a implantar en algunos centros penitenciarios el Programa "Control de la agresión sexual" (SAC) cuyos objetivos son:

a. El reconocimiento del penado de las situaciones de riesgo que le han conducido a la comisión del delito:

b. El aprendizaje del control de impulsos;

c. La concientización por parte del recluso de los daños producidos a las víctimas, sus familiares y personas de su entorno; y

d. La asunción de responsabilidad de sus actos y de las consecuencias derivadas de ello.

Las características del programa son: a) La orientación cognitivo-conductual, que sigue el modelo de "Prevención de la recaída"; b) Está destinado a agresores sexuales con al menos dos víctimas en su haber; c) No está condicionado a la obtención de beneficios penitenciarios en sentido amplio (permisos de salida, tercer grado, libertad condicional, etc.) y es llevado a cabo en coordinación con las autoridades judiciales en pro de prevenir contingencias que menoscaben el proceso de tratamiento; d) Se intenta seleccionar a agresores que se encuentren en el último tramo de su condena (tres o cuatro años); e) Las sesiones terapéuticas son preferentemente grupales; f) Hay tres áreas principales de intervención: conducta sexual, funcionamiento, competencia social y distorsiones cognitivas; g) No se esperan resultados espectaculares y se dirige a reducir el riesgo de reincidencia; y h) Es aconsejable su continuación en el medio libre una vez recuperada la libertad.

En lo específico, el programa SAC tiene como antecedentes inmediatos investigaciones sobre delincuentes sexuales desarrollados entre 1993 y 1995 en las cárceles españolas (Garrido, Redondo y otros, 1995) y que posibilitaron a partir de 1996 a Garrido y Baneyto proponer un primer programa estructurado, intensivo e integral de tratamiento de agresores sexuales en España. La primera aplicación de este programa se llevó a cabo paralelamente en dos centros penitenciarios de la provincia de Barcelona: Quatre Camins y Brians (Roca y Montero, 2000). Este mismo programa, con algunas adaptaciones, se aplica en la actualidad en diversos centros penitenciarios españoles.

Los elementos distintivos del programa se establecen fundamentalmente en torno a los supuestos y características que adopta, los que se describen en detalle en el manual del terapeuta del programa SAC (Ministerio del Interior de España, 2006). En lo que refiere a los supuestos centrales, estos serían los siguientes (p. 18-23):

Supuesto $\mathrm{N}^{\mathrm{o}} 1$ : Sin un tratamiento psicológico especialmente eficaz y adecuado, las agresiones sexuales se repetirán y se convertirán en una constante que supone elevado riesgo para la sociedad.

Supuesto $\mathrm{N}^{\circ}$ 2: El interés sexual desviado puede conceptualizarse como un auténtico proceso de adicción. Tales patrones de adicción no pueden ser superados en general sólo por la mera estancia en prisión o el paso del tiempo. Sin tratamiento adecuado, el delincuente sexual reincidirá ante cualquier factor de riesgo.

Supuesto $\mathrm{N}^{\circ}$ 3: La sola participación en el programa no debe considerarse como un requisito suficiente para adoptar decisiones sobre progresión de grados en la condena o acceso a libertad condicional, por lo que se deberá valorizar en forma individual, y caso a caso, los factores de riesgo involucrados.

En lo relacionado con las características fundamentales del programa, estas se describen a continuación: 
Característica $\mathrm{N}^{\circ} \mathbf{1}$ : El programa contempla dos fases claramente diferenciadas, y que considera una evaluación en profundidad de cada sujeto que participa voluntariamente (en dimensiones tales como conducta sexual, el funcionamiento social y las distorsiones cognitivas) y una etapa de intervención psicosocial intensiva.

Característica $\mathrm{N}^{\circ}$ 2: Los fundamentos de acción se basan en la propuesta conceptual y empírica de Marshall y Barbaree (1989), los patrones cognitivos predelictivos en el modelo de estilo de vida criminal de G.D. Walters (1989) y en la estructura de prevención de recaída de Pithers $(1987,1988)$. Estas orientaciones se expresan en un programa que presenta una distribución modular y una secuencialización de las técnicas aplicadas.

Característica No3: La participación en el programa es voluntaria y los sujetos que desean participar deben cumplir los siguientes requisitos: (a) ser varón, (b) cumplir condena por un delito sexual o más, (c) estar próximo a la libertad condicional o al cumplimiento de la condena, (d) tener entre 18 y 60 años, (e) no tener causas pendientes, (f) poseer un cuociente intelectual superior a $80 \mathrm{y}$ tener conocimiento básico de lectoescritura, (g) No sufrir enfermedad mental que pueda alterar su funcionamiento en el programa, y ( $h$ ) no padecer enfermedades físicas que puedan repercutir en la participación en el programa.

Los contenidos específicos del programa se distribuyen en nueve módulos psicoeducativos y terapéuticos, que consideran elementos tales como (Camps, p.9): (a) conciencia emocional, (b) empatía hacia la víctima, (c) mecanismos de defensa, (d) distorsiones cognitivas, (e) autocontrol, (f) educación afectivo-sexual, (g) resolución de problemas, (h) habilidades sociales y asertividad, e (i) desarrollo de valores y prevención de recaídas.

En la actualidad, los centros penitenciarios españoles que cuentan en España con módulos o unidades de vida específicas para el tratamiento de la violencia sexual son el QP QuatreCamins, Brians 1 y Brians 2.

La aplicación sistemática del programa SAC ha cumplido más de un decenio en las prisiones españolas, posibilitando contar con una serie de antecedentes relativos a su grado de efectividad e impacto sobre la reincidencia delictiva. En relación con este aspecto, la revisión de la literatura disponible no permite establecer una tendencia unívoca de los resultados, adoptando un carácter controversial, ya que mientras algunos autores establecen que el programa muestra evidencia empírica positiva en la reducción de la reincidencia delictiva, en torno a tasas que bordean el 14\% (Redondo, 2006; 2008), otros argumentan que "los programas específicos de tratamiento de orientación cognitivo-conductual no tienen efectos significativos respecto de otras actividades que los internos realizan en la mejora de su adaptación personal" (Medina García, 2013).

Al menos en forma preliminar, uno de los aspectos centrales de la controversia en torno a la determinación de las tasas de reducción de la reincidencia se sitúa en torno a la consideración del efecto de maduración de los sujetos evaluados, así como al empleo y selección de un grupo control, para la determinación de las diferencias significativas entre grupos y poder determinar el tamaño del efecto.

\section{Experiencia en Chile}

Gendarmería de Chile aplica el modelo de Riesgo -Necesidad- Responsividad. (Risk -Need- Responsivity) El progreso en el conocimiento sobre materias de rehabilitación y reinserción social ha permitido establecer que los programas de tratamiento para infractores de ley pueden lograr resultados favorables, no sólo desde la perspectiva de colaborar con la integración social de las personas usuarias de estos programas, sino que también pueden generar reducciones en la reincidencia en delito en esta población. Múltiples evidencias han permitido determinar los principios básicos que orientan los proyectos de intervención que logran buenos resultados en términos de disminución de la reincidencia en delito (Bourgon y Armstrong, 2005).

Según Gendarmería de Chile, la síntesis de los principios que determinan el éxito de la intervención se ha resumido en el modelo de Riesgo - Necesidad - Responsividad, cuyas características principales se reseñan a continuación:

El Principio de Riesgo establece que el nivel de tratamiento que recibe un infractor de ley debe ajustarse al nivel de riesgo de éste último. Esto es, infractores de mayor riesgo requieren niveles intensivos de tratamiento, mientras que los infractores de más bajo riesgo requieren niveles mínimos de intervención, lo cual supone contar con la capacidad para evaluar el riesgo de manera confiable, no sólo como estrategia de monitoreo y seguimiento de casos, sino también para la toma de decisiones respecto a la derivación a la medida de tratamiento más eficaz para cada caso.

El Principio de Necesidad identifica dos grandes tipos de necesidades en el sujeto infractor: las 
criminogénicas (estáticas y dinámicas) y las no criminogénicas. Las necesidades criminogénicas son factores de riesgo que, al ser modificados, se asocian con cambios en la reincidencia. El tratamiento efectivo del infractor de ley será aquel que se enfoque fundamentalmente en este tipo de necesidades. Por ejemplo, el abuso de sustancias y los problemas laborales son necesidades criminogénicas que deben ser abordadas por la intervención; en tanto que necesidades no criminogénicas como la ansiedad y la autoestima deben limitarse a un rol secundario, dado que focalizar la intervención sobre estos aspectos no impacta sobre el comportamiento infractor en el futuro.

El Principio de Responsividad (disposición a responder) establece que existen ciertas características cognitivo-conductuales y de personalidad en los infractores de ley que influencian su disposición a responder ante diversos tipos de tratamiento. En general, las intervenciones de carácter cognitivoconductual u orientadas al aprendizaje social logran mejores resultados que otras estrategias de tratamiento (responsividad general). Sin perjuicio de ello, la manera en que se dispongan las acciones de tratamiento debe considerar los estilos de aprendizaje y motivaciones de las personas que participan de la intervención (responsividad específica).

El Principio de Discrecionalidad Profesional señala que algunos infractores de ley pueden presentar características o situaciones particulares que no son adecuadamente consideradas por los otros principios. Por ejemplo, los agresores sexuales que presenten un buen ajuste social y normativo podrían ponderar un bajo nivel de riesgo en instrumentos estandarizados de evaluación; sin embargo, los profesionales que atienden estos casos podrían detectar otras condiciones de riesgo, tales como la búsqueda selectiva de empleos por parte del infractor que le permitan acercarse a víctimas potenciales. En Chile, la existencia de programas de tratamientos especializados orientados a la intervención de agresores sexuales inimputables; infractores de Ley Penal Juvenil y población penal adulta es escasa.

A nivel del SENAME (Servicio Nacional de Menores), podemos mencionar el programa especializado para "niños/as y adolescentes que presentan conductas abusivas de carácter sexual" (PAS); oferta programática que se fundamenta en resultados de la investigación internacional. Esta señala que alrededor del 20\% de las violaciones y 50\% de abusos han sido realizados por adolescentes y que aproximadamente el 50\% de los adultos con conductas de ofensa sexual han cometido las primeras agresiones durante su juventud. (Díaz Morfa, 2003; Save the Children, 2000). Los estudios también plantean que el tratamiento temprano previene la cronificación del comportamiento abusivo, que es altamente autorreforzante y difícil de modificar una vez instaurado (Jones Vernon, Save the Children, 2000).

En el sistema de cumplimiento de condena abierto de Gendarmería de Chile (CRS) podemos señalar la experiencia del Programa PIDOS aplicado a agresores sexuales adultos que cumplen condenas en el medio libre bajo medida alternativa de libertad vigilada. Este programa pretende generar nuevas herramientas que contribuyan a la reinserción social de aquellas personas que han cometido delitos sexuales y que se encuentran bajo control y supervisión en la libertad vigilada del adulto. El objetivo general es disminuir la ocurrencia de comportamientos de violencia sexual en agresores sexuales condenados a la libertad vigilada del adulto (Gendarmería de Chile, 2012).

Otra experiencia existente en el sistema de cumplimiento de condena abierto (CRS de Temuco) es la aplicación del modelo español SAC, que, como ya se señaló precedentemente, se basa en el reconocimiento de las situaciones de riesgo, el aprendizaje del control de impulsos, la concientización de los daños causados, la asunción de la responsabilidad y las consecuencias de sus actos.

En el sistema de cumplimiento de condena cerrado (CCP) es más probable encontrar acciones de prevención secundaria y terciaria centrada en la víctima, pero la prevención terciaria que se focaliza en el agresor es casi inexistente. Este hecho deja abierta la posibilidad de reincidencia al no entregar una oferta de servicios durante la condena o de una eventual oferta de apoyo con posterioridad a su cumplimiento. Desde esta perspectiva, la intervención terciaria contribuye a la seguridad de los ciudadanos cuando la condena ya se ha cumplido. Al examinar la situación se constata que no se cuenta con programas diseñados especialmente para atender a los condenados por delitos sexuales, situación que explica el que no se tengan datos sobre los resultados de la condena en estos delitos (Macchino, 2014).

Este hecho releva la necesidad de contar con una intervención especializada y focalizada en este tipo de internos, dado que las características que presentan este tipo de agresores les otorga un carácter distintivo respecto de otro tipo de población delictual. 


\section{Conclusión}

El tiempo de condena no necesariamente tiene concordancia con la efectividad de la pena. Esto debido a que mientras no sean modificados los factores que desencadenan la conducta criminal, es probable que estos se mantengan inalterados. Esto pone de relieve la necesidad de intervención a los agresores sexuales, a fin de que estos logren en alguna medida un nivel de control de los factores que precipitan o desencadenan la conducta delictiva.

Esto cobra relevancia aun cuando la población de agresores sexuales estadísticamente hablando es significativamente inferior a la de la población carcelaria general, atendiendo a que el impacto y daño social que genera la comisión de delitos sexuales en la sociedad es alto.

Pese a lo anterior, y a la existencia de programas de intervención psicosocial especializados en agresores sexuales a nivel internacional, la realidad chilena evidencia que, en general, estos no han sido suficientemente acogidos y aplicados en nuestro sistema carcelario. De este modo, es posible concluir que en el contexto nacional, la aplicación de programas orientados al control de la agresión sexual y disminución de la reincidencia delictiva resultan escasos y asistemáticos.

Una posible explicación a considerar la constituye la falta de una política criminal clara en materia de delitos sexuales, orientada a la disminución de la reincidencia por un lado, y por otro al logro de un control de los factores que provocan la conducta delictiva de carácter sexual. Esta situación se traduce en la práctica en que internos agresores sexuales del sistema cerrado (debido a falta de intervención especializada y con independencia de sus características individuales y riesgo de reincidencia) puedan acceder a beneficios intra y extrapenitenciarios con mayor facilidad, dado que sus características los predisponen favorablemente al cumplimiento de conducta reglamentaria para optar a estos.

Finalmente, la falta de aplicación y adaptación de modelos, lineamientos de intervención e instrumentos estandarizados a nivel nacional impactan negativamente sobre los equipos técnicos, los que deben responder a un reglamento interno indiferenciado, que regula el otorgamiento de los beneficios sin distinción entre agresores sexuales y otro tipo de población delictual.

\section{Bibliografía}

- BERLIN, F.S. (2000) The etiology and treatment of sexual offending. En D. Fiscbein: The Science, Treatment and Prevention of Antisocial Behaviors (Cap.21). Kingston, New Jersey: Civic Research Institute.

- BROWN, S. (2005) Treating sex offender. Cullompton, Devon (UK): William Publishing

- Fiscalia NACIONAL (2013) Cuenta Pública Fiscal Nacional Sr. Sabas Chahuán Sarrás. Disponible en http://www.fiscaliadechile.cl/cuentapublica2013/cuenta_publica_2013.pdf Consultado el 15 de septiembre de 2014 .

- ECHEbARÚA, E. \& GUERRICAECHEVARRÍA, C. (2000) Abuso sexual en la infancia: Víctima y agresores. Un enfoque clínico. Ed. Ariel, Barcelona, España.

- GARRIDO, V. \&BENEYTO, M.J. (1996) El control de la agresión sexual. Un programa de tratamiento para delincuentes sexuales en prisión y en la comunidad. Valencia: Ed. Cristobal Serrano Villalba

- GARRIDO, V. \& BENEYTO, M.J. (1996) Els delinquents sexuals institucionalitzats. Un estudi del agressors de nens. Barcelona: Centre d’Estudis Juridics i Formació Especialitzada.

- GARRIDO, V. \& BENEYTO M.J. (1997) La valoración psicológica de los agresores sexuales: los delitos, la reincidencia y el tratamiento. En Cuadernos de Derecho Judicial: Delitos contra la libertad sexual. Escuela Judicial. Consejo General del Poder Judicial.

- GUILlamONDEGUI, L.: (2006) Necesidad de programas de políticas públicas-privadas preventivas y de tratamiento. ¿La resocialización de los delincuentes sexuales es posible? Una perspectiva comparada. Trabajo publicado en ICARO. Revista de ejecución de la pena privativa de la libertad y el encierro, Año $1, N^{0} 1$, Fabián Di Plácido Editor, Buenos Aires, 2006, pp. 225251.

- INSTITUTO DE ASUNTOS PUBLICOS U. DE CHILE (2010). Debates Penitenciarios, Revista Electrónica Nº 13, Área de Estudios Penitenciarios CESC.

- LEGANEZ GOMEZ en El control de la agresión sexual. Un programa de tratamiento para delincuentes sexuales en prisión y en la comunidad. Manual para el terapeuta. Ed. Cristóbal Serrano Villalba, pp 97-99. Año 2006

- LOVINS, BRIAN, LOWENKAMP, CHRISTOPHER Y LATESSA, EDWARD (2009). Applying the risk principle to sex offenders: Can treatment make some sex offenders worse? ThePrisonjournal, Vol. 89, No 3, pp. 344-357.

- MARSHALL, W.L. \& BARBAREE, H.E. (1989) Sexual violence. EnK.Howells y C.R. Hollins (Eds), Clinical Approches to Violence.Chinchester: John Wiley and Sons. 
- MARSHALL, W.L. \& REDONDO, S. (2002) Control y tratamiento de la agresión sexual. En S. Redondo (Coord.) Delincuencia sexual y sociedad (págs. 301328). Ed. Ariel, Barcelona.

- MARSHALL, W.L. \&SERRAN, G.A. (2004) Tratamiento del agresor sexual. En J. Sanmartín (Coord.) El laberinto de la violencia ((págs. 309-319). Ed. Ariel, Barcelona.

- MEDiNA, P. (2013) Evaluación experimental de la eficacia de los programas psicológicos de tratamiento penitenciario. Gobierno de España, Ministerio del Interior, Secretaría general de Instituciones Penitenciarias. Premio Nacional Victoria Kent.

- NAVARRO, J.C. (2004) El tratamiento de los agresores sexuales en prisión. I Congreso de Psicología Jurídica y Forense en Red, 3-14 de mayo de 2004.

- PÉREZ, M.; REDONDO, S.; MARTÍNEZ, M.; FORERO, C.; PUEYO, A. (2008) Predicción de riesgo de reincidencia en agresores sexuales. Revista Psicothema. Vol. 20, No 2, pp.205-2010.

- REDONDO, S.; SANCHEZ-MECA, J.; GARRIDO, V. (2000b) Los programas psicológicos con delincuentes y su efectividad: La situación europea. Psicothema, 14 (Supl.) 164-173

- REDONDO, S. (2006) ¿Sirve el tratamiento para rehabilitar a los delincuentes sexuales? Revista Española de Investigación Criminológica. Artículo 6, No4, pp.1-22.

- REDONDO, S. \&GARRIDO, V. (2008) Efficacy of a psychological treatment for sex offenders. Psicothema. Vol. 20, Nº1, pp. 4-9.

- ROCA, M. \& MONTERO, P. (2000) Intervención con psicópatas en prisión. En A. Raine y J. Sanmartin: Violencia y psicopatía (Estudios sobre violencia, $N^{\circ} 4$ ). Ed. Ariel/Centro Reina Sofía para el estudio de la Violencia. Barcelona, España.

- MINISTERIO DEL INTERIOR ESPAÑA (2006) El control de la agresión sexual. Programa de intervención en el medio penitenciario. Un programa de tratamiento para delincuentes sexuales en prisión. Documentos penitenciarios 3, Dirección General de Instituciones Penitenciarias. 
